\title{
铜催化萗胺分子内去芳构化芳基化反应
}

\author{
芦军梁仁校贾义霞* \\ (浙江工业大学化工学院 杭州 310014)
}

\begin{abstract}
摘要 发展了碘化亚铜催化的芳基碘化物与荎胺的分子内 Ullmann 类型偶联反应，实现了䒺胺分子内去芳构化芳基化 反应，以良好至优异的收率合成了一系列 $3 H$-吲哚衍生物.
\end{abstract}

关键词 Ullmann 偶联; 萗胺; 芳基化; 去芳构化

\section{Copper-Catalyzed Intramolecular Dearomative Arylation of Naphthylamines}

\author{
Lu, Jun Liang, Renxiao Jia, Yixia* \\ (College of Chemical Engineering, Zhejiang University of Technology, Hangzhou 310014)
}

\begin{abstract}
A CuI-catalyzed intramolecular dearomative arylation of naphthylamines is developed through a Ullmann-type cross-coupling reaction, which led to a range of fused $3 H$-indole derivatives in good to excellent yields.

Keywords Ullmann coupling; naphthylamine; arylation; dearomatization
\end{abstract}

近年来，过渡金属催化的芳香化合物去芳构化反应 已成为非芳香环状化合物构筑的重要方法, 并被应用于 天然产物及生物活性分子的合成中 ${ }^{[1]}$. 苯酚、苯胺及 $\mathrm{N}-\mathrm{H}$ 吲哚或吡咯等芳香化合物基于酮一烯醇互变异构, 利用过渡金属催化的交叉偶联反应, 实现了与卤代芳 烃、烯丙醇酯等亲电试剂的交叉偶联去芳构化反应 ${ }^{[2-4]}$. 此外, 利用迁移插入策略, $N$-取代吲哚、吡咯、苯并呋喃、 呋喃以及菜等芳香化合物环内碳一碳双键参与的新型 Heck 反应及其串联反应也得到了快速发展, 成为重要 的去芳构化反应方法 ${ }^{[5]}$. 尽管如此, 目前上述去芳构化 反应使用的过渡金属催化剂主要为钯、铱等贵金属. 相 比，铜、镍等廉价金属在去芳构化反应中的应用报道较 少. 显然, 发展价格低廉、低毒甚至无毒的过渡金属催 化的去芳构化反应, 实现杂环化合物的高效合成, 具有 重要的学术价值和应用前景.

铜催化的 Ullmann 偶联反应可高效构建碳一氮键和 碳一氧 键 $^{[6]}$. 相比较, 芳基卤代烃与碳基亲核试剂的 Ullmann 类交叉偶联反应构建碳一碳键的研究仍较少. 2007 至 2010 年, Jiang, Cacchi 及 Bach 等研究小组先后
报道了铜催化碘代芳烃与烯胺酮的分子内 $\mathrm{C}-\mathrm{C}$ 键偶联 反应，实现了吲哚衍生物的快捷合成(图 1a) ${ }^{[7]} .2009$ 年, Buchwald 小组 ${ }^{[8]}$ 利用手性钯催化剂, 实现了菜胺分子内 不对称去芳构化芳基化反应，合成了一系列手性 $3 H$-吲 哚类化合物. 受上述工作启发, 本工作组以廉价金属铜 为催化剂, 研究卤代芳烃与荎胺的分子内交叉偶联去芳 构化反应，构建 $3 H$-吲哚衍生物 ${ }^{[9]}$.

(a) Previous work: Cu-catalyzed intramolecular arylation of enamines

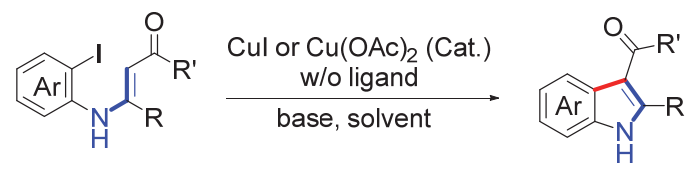

(b) This work: Cul-catalyzed dearomative arylation of naphthylamines

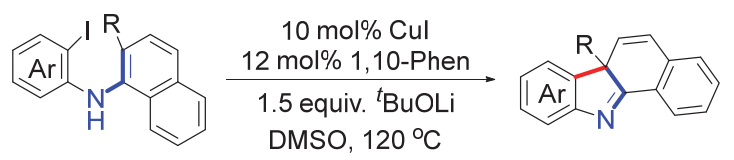

图 1 铜催化烯胺分子内芳基化及萗胺去芳构化芳基化反应 Figure $1 \mathrm{Cu}$-catalyzed intramolecular enamine arylation and dearomative arylation of naphthylamines

\footnotetext{
* Corresponding author. E-mail: yxjia@zjut.edu.cn

Received May 29, 2021; revised July 31, 2021; published online August 17, 2021.

Dedicated to the 100th anniversary of Chemistry at Nankai University.

Project supported by the National Natural Science Foundation of China (Nos. 21702184, 22071217, 91956117).

国家自然科学基金(Nos. 21702184, 22071217, 91956117)资助项目.
} 


\section{1 结果与讨论}

以 $N$-邻碘苯基-1-萘胺(1a)为模型底物研究分子内 去芳构化芳基化反应，结果见表 1 . 在 $\mathrm{CuI}$ 催化下，以 1,10-菲罗啉为配体, 叔丁醇锂为碱, 底物 $1 \mathbf{a}$ 在 $100{ }^{\circ} \mathrm{C}$ 下四氢呋喃溶液中反应 $36 \mathrm{~h}$, 以 $20 \%$ 的收率获得去芳构 化产物 2a(表 1, Entry 1). 受此鼓舞, 首先对溶剂效应进 行了考察, 发现溶剂对产物收率有明显影响. 当反应在 1,4-二氧六环溶剂中进行时, 产物 $\mathbf{2 a}$ 的收率提高至 50\%(表 1, Entry 2), 而在非极性溶剂甲苯中收率仅为 $23 \%$ (表 1, Entry 3). 令人高兴的是, 极性非质子溶剂二 甲基亚砜对反应有显著的促进作用, 产物 $\mathbf{2 a}$ 的收率可 达 92\%(表 1, Entry 4). 随后，在二甲基亚砜溶剂中对碱 进行了考察. 反应在有机碱三乙胺存在时不能发生, 而 在无机碱磷酸钾和碳酸钾下分别以 $85 \%$ 和 $89 \%$ 的收率 获得目标产物(表 1, Entries 5～7). 配体实验表明 1,10菲罗啉是较优配体, 以卡宾 IMes 及 $L$-脯氨酸为配体时 产物收率分别为 27\% 和 29\%(表 1, Entries 8,9), 而无配 体参与时产物收率为 25\%(表 1, Entry 10). 此外, 与碘化 物相比, 以芳基溴化物为底物时目标产物的收率降低至 66\%(表 1, Entry 11). 当使用氯化亚铜为催化剂时, 反应 收率为 $72 \%$ (表 1, Entry 12), 而无铜催化剂时不发生反 应(表 1, Entry 13), 说明碘化亚铜催化剂在反应中起关 键作用. 基于上述对催化剂、溶剂、配体、碱的笁选, 我 们确定了较优的反应条件为: 以 $10 \mathrm{~mol} \% \mathrm{CuI}$ 为催化剂,

表 1 反应条件优化 ${ }^{a}$

Table 1 Condition optimization
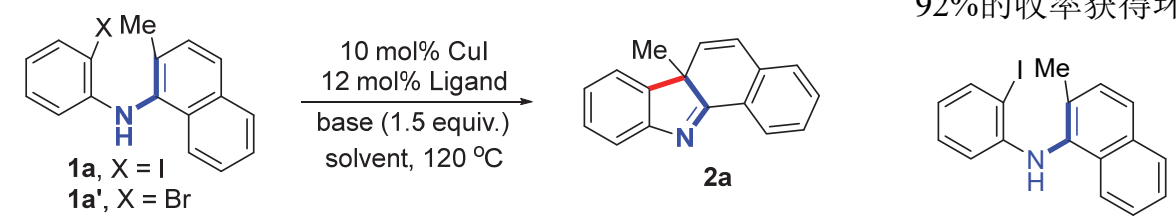

$1 a^{\prime}, X=B r$

\begin{tabular}{clclc}
\hline Entry & Solvent & Base & Ligand & Yield $^{b} \%$ \\
\hline 1 & THF & ${ }^{t} \mathrm{BuOLi}$ & 1,10 -Phen & 20 \\
2 & Dioxane & ${ }^{t} \mathrm{BuOLi}$ & 1,10 -Phen & 50 \\
3 & Toluene & ${ }^{t} \mathrm{BuOLi}$ & 1,10 -Phen & 23 \\
4 & DMSO & ${ }^{t} \mathrm{BuOLi}$ & 1,10 -Phen & 92 \\
5 & DMSO & $\mathrm{Et}_{3} \mathrm{~N}$ & 1,10 -Phen & $\mathrm{NR}$ \\
6 & DMSO & $\mathrm{K}_{3} \mathrm{PO}_{4}$ & 1,10 -Phen & 85 \\
7 & DMSO & $\mathrm{K}_{2} \mathrm{CO}_{3}$ & 1,10 -Phen & 89 \\
8 & DMSO & ${ }^{t} \mathrm{BuOLi}$ & IMes & 27 \\
9 & DMSO & ${ }^{t} \mathrm{BuOLi}$ & $L$-Proline & 29 \\
10 & DMSO & ${ }^{t} \mathrm{BuOLi}$ & - & 25 \\
$11^{c}$ & DMSO & ${ }^{t} \mathrm{BuOLi}$ & $1,10-P h e n$ & 66 \\
$12^{d}$ & DMSO & ${ }^{t} \mathrm{BuOLi}$ & $1,10-P h e n$ & 72 \\
$13^{e}$ & DMSO & ${ }^{t} \mathrm{BuOLi}$ & 1,10 -Phen & $\mathrm{NR}$ \\
\hline
\end{tabular}

${ }^{a}$ Reaction conditions: 1a $(0.2 \mathrm{mmol}), \mathrm{CuI}(0.02 \mathrm{mmol})$, ligand $(0.024 \mathrm{mmol})$, and base $(0.3 \mathrm{mmol})$ in solvent at $120{ }^{\circ} \mathrm{C}$ for $36 \mathrm{~h} .{ }^{b}$ Isolated yield. ${ }^{c} \mathbf{1 a}(0.2$ mmol) was used instead of 1a. ${ }^{d} 0.02 \mathrm{mmol} \mathrm{CuCl}$ was used instead of $\mathrm{CuI}$. ${ }^{e}$ Without CuI.
$12 \mathrm{~mol} \%$ 1,10-菲罗啉为配体, 1.5 equiv. ${ }^{t} \mathrm{BuOLi}$ 为碱，在 二甲基亚砜溶剂中 $120^{\circ} \mathrm{C}$ 下反应 $36 \mathrm{~h}$.

获得上述较优条件后，对反应底物普适性进行了考 察. 如表 2 所示, 首先对䒺环胺基邻位 $\mathrm{R}$ 基团的取代基 效应进行了探索. 当取代基为甲基、乙基或苯基时，反 应以良好至优异的收率获得目标产物 $\mathbf{2 a} \sim \mathbf{2 c}$, 其中苯 基取代的产物 $2 \mathrm{c}$ 的收率略低(83\%). 随后考察了一系列 芳香取代基对反应的影响. 研究发现无论是给电子基 $(\mathbf{2 d}, 2 \mathrm{e})$ 还是吸电子基 $(\mathbf{2 f}, \mathbf{2 g})$, 相应的反应都获得良好 至优异的收率. 此外, 具有一定位阻效应的邻甲基(2h) 和 3,5-二甲基(2i)底物也取得良好的收率. 进一步, 䒺环 胺基邻位的杂芳基呋喃及稠环 2-菜基也能良好地兼容, 分别以 $68 \%$ 和 $89 \%$ 的收率获得相应的去芳构化芳基化 产物 $2 \mathbf{j}$ 和 $2 \mathbf{k}$. 随后对菜环胺基 4-位的取代基也进行了 考察，发现一系列芳基(如对甲苯基、对氯苯基、苯基等) 均能良好地兼容，以良好至优异的收率( $85 \%$ ～96\%)获 得相应的目标产物 2l 20. 最后, 对碘代芳基环上的取 代基进行了简单的拓展，分别以 74\%和 48\%获得相应的 目标产物 $2 \mathbf{p}$ 和 $2 \mathbf{q}$.

完成上述研究后，我们对反应产物进行了简单的合 成转化. 如图 2 所示, 克级规模实验表明 $3.0 \mathrm{mmol}$ 的标 准底物 $1 \mathrm{a}$ 在较优反应条件下可以 $89 \%$ 的收率获得产物 2a. 产物 2c 与 $N$-溴代丁二酰亚胺(NBS) 在四氢呋喃和水 的混合溶液中室温下反应，可以 $86 \%$ 的收率生成烯烃溴 化着基化产物 3 . 化合物 3 经叔丁醇钾处理, 在叔丁醇 和四氢呋喃的混合溶液中发生分子内亲核取代反应，以 92\%的收率获得环氧化合物 4 .

$1 \mathrm{a}(1.08 \mathrm{~g}, 3.0 \mathrm{mmol})$
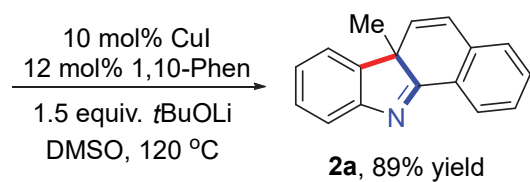

$2 a, 89 \%$ yield

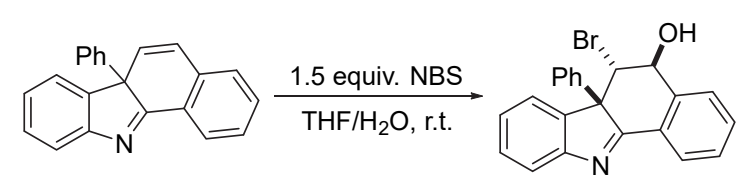

2c

$3,86 \%$ yield

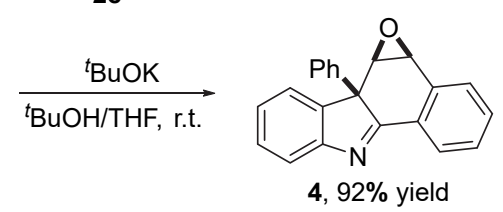

图 2 克级规模反应及产物合成转化

Figure 2 Gram-scale reaction and synthetic transformation of the product

基于文献研究我们提出了如下可能的反应机理 ${ }^{[7]}$. 如图 3 所示, 首先在碱的作用下苯胺与铜催化剂形成 
表 2 菜胺去芳构化反应底物拓展 ${ }^{a}$

Table 2 Substrate scope of naphthylamine dearomatization

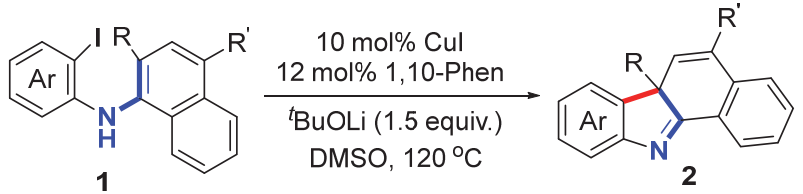

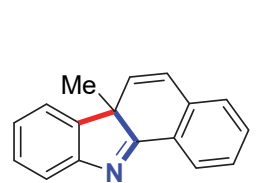

2a, $92 \%$

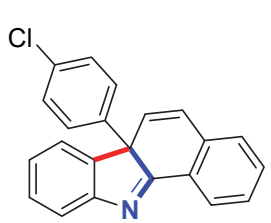

$2 \mathrm{~g}, 88 \%$

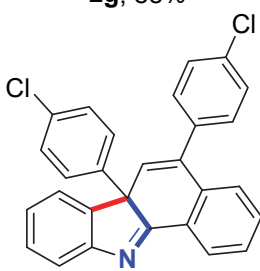

$2 \mathrm{~m}, 85 \%$

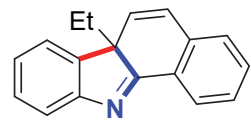

2b, $90 \%$

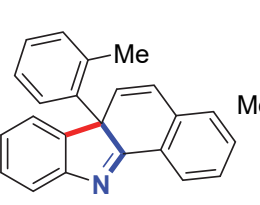

2h, $85 \%$

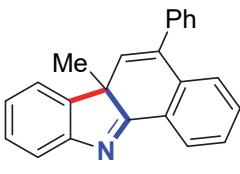

2n, $94 \%$

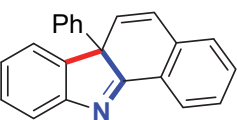

2c, $83 \%$

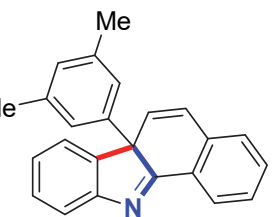

$\mathbf{2 i}, 83 \%$

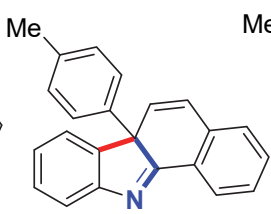

2d, $90 \%$
$\mathrm{MeO}$

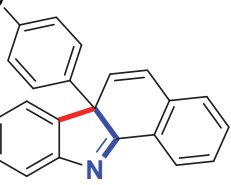

$2 e, 85 \%$

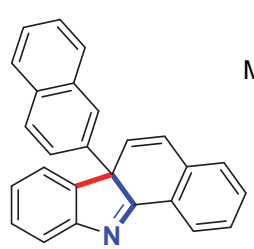

2k, $89 \%$

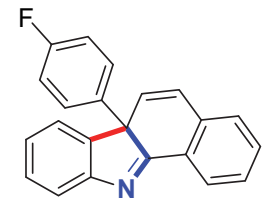

2f, $84 \%$

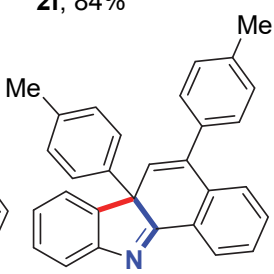

2I, $91 \%$

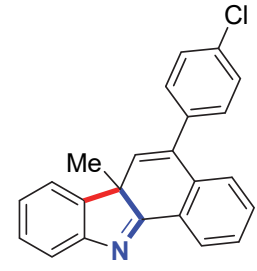

2o, $96 \%$

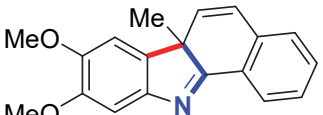

2p, $74 \%$

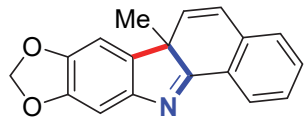

$2 q, 48 \%$

${ }^{a}$ Reaction condition: $1(0.2 \mathrm{mmol}), \mathrm{CuI}(0.02 \mathrm{mmol}), 1,10$-phen $(0.024 \mathrm{mmol})$, and ${ }^{t} \mathrm{BuOLi}(0.3 \mathrm{mmol})$ in $\mathrm{DMSO}$ at $120{ }^{\circ} \mathrm{C}$ for $36 \mathrm{~h}$.

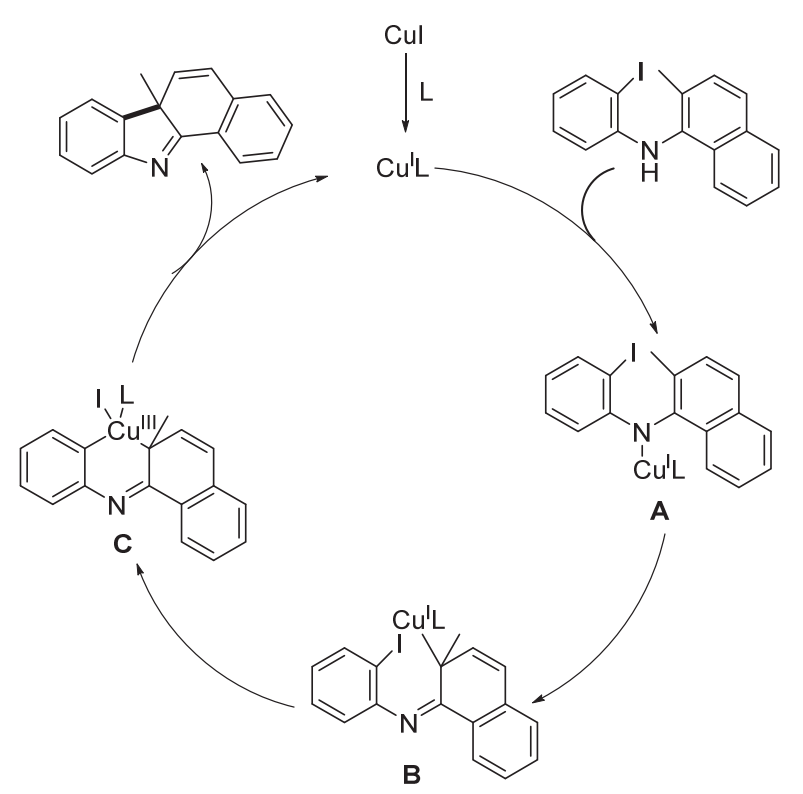

图 3 荎胺去芳构化反应的建议机理

Figure 3 Proposed mechanism of naphthylamine dearomatization

$\mathrm{N}-\mathrm{Cu}$ 中间体 $\mathbf{A}$. 随后 $\mathrm{N}-\mathrm{Cu}$ 键异构为 $\mathrm{C}-\mathrm{Cu}$ 键形成 中间体 $\mathbf{B}, \mathbf{B}$ 与芳基碘化物发生分子内氧化加成形成三
价铜中间体 C. 最后，经还原消除得到目标产物并释放 一价铜催化剂, 完成催化循环过程.

\section{2 结论}

以碘化亚铜为催化剂, 通过芳基碘化物与萘胺的分 子内 Ullmann 类型偶联反应，实现了䒬胺分子内去芳构 化芳基化反应. 该催化反应具有良好的底物普适性和官 能团兼容性，并以良好至优异的收率高效合成了一系列 $3 H$-吲哚衍生物.

\section{3 实验部分}

\section{1 仪器与试剂}

实验所用溶剂使用前均按照处理溶剂的标准方法 进行. ${ }^{1} \mathrm{H}$ NMR, ${ }^{13} \mathrm{C}$ NMR 均在 Bruker AVANCE III 500 $\mathrm{MHz}$ 型核磁共振仪上测定，溶剂 $\mathrm{CDCl}_{3}$ ，内标 TMS; 柱 层析使用 $200 \sim 300$ 目硅胶，展开剂为石油醚 $(60 \sim$ $\left.90{ }^{\circ} \mathrm{C}\right)$ 和乙酸乙酯. 所有实验药品均为市售分析纯试 剂.

\section{2 实验方法}

反应的通用方法: 在 Schlenk 管中依次加入底物 1 
(0.2 mmol)、 $10 \mathrm{~mol} \%$ 碘化亚铜、 $12 \mathrm{~mol} \%$ 1,10-菲咯啉和 1.5 equiv. 叔丁醇锂. 氮气置换三次后, 通过注射器加入 $2.0 \mathrm{~mL}$ 无水二甲基亚砜溶剂. 反应在 $120{ }^{\circ} \mathrm{C}$ 反应 $36 \mathrm{~h}$. 反应完成后冷却至室温, 反应液经水淬灭后以乙酸乙酯 萃取后, 饱和食盐水洗涤三次. 随后收集有机相, 无水 硫酸钠干燥后旋转蒸发仪除去溶剂, 通过柱层析分离得 到产物 [淋洗液: $V$ (石油醚) $: V($ 乙酸乙酯 $)=20 ： 1$ ].

$6 \mathrm{a}$-甲基- $6 \mathrm{a} H$-苯并 $[a]$ 咔唑 $(\mathbf{2} \mathbf{a})$ ：黄色油状物，产率 92\%. ${ }^{1} \mathrm{H}$ NMR (500 MHz, $\left.\mathrm{CDCl}_{3}\right) \delta: 8.04(\mathrm{~d}, J=6.5 \mathrm{~Hz}$, $1 \mathrm{H}), 7.73(\mathrm{~d}, J=7.5 \mathrm{~Hz}, 1 \mathrm{H}), 7.43 \sim 7.40(\mathrm{~m}, 2 \mathrm{H}), 7.37(\mathrm{t}$, $J=7.0 \mathrm{~Hz}, 2 \mathrm{H}), 7.23$ (t, $J=8.0 \mathrm{~Hz}, 1 \mathrm{H}), 7.19$ (d, $J=7.5$ $\mathrm{Hz}, 1 \mathrm{H}), 6.51$ (d, $J=9.5 \mathrm{~Hz}, 1 \mathrm{H}), 6.41(\mathrm{~d}, J=9.5 \mathrm{~Hz}, 1 \mathrm{H})$, 1.44 (s, 3H); ${ }^{13} \mathrm{C}$ NMR (125 MHz, $\left.\mathrm{CDCl}_{3}\right) \delta: 185.3,154.4$, $141.7,136.4,133.7,131.5,128.9,128.3,127.9,127.4$, 126.6, 125.5, 125.1, 121.4, 121.3, 57.0, 27.9; HRMS (ESI) calcd for $\mathrm{C}_{17} \mathrm{H}_{14} \mathrm{~N}[\mathrm{M}+\mathrm{H}]^{+}$232.1121, found 232.1128.

$6 \mathrm{a}$-乙基- $6 \mathrm{a} H$-苯并 $[a]$ 咔唑 $(\mathbf{2 b})$ ：黄色油状物, 产率 $90 \% .{ }^{1} \mathrm{H}$ NMR (500 MHz, $\left.\mathrm{CDCl}_{3}\right) \delta: 8.01 \sim 8.00(\mathrm{~m}, 1 \mathrm{H})$, $7.70(\mathrm{~d}, J=8 \mathrm{~Hz}, 1 \mathrm{H}), 7.46 \sim 7.42(\mathrm{~m}, 2 \mathrm{H}), 7.41 \sim 7.36(\mathrm{~m}$, 2H), $7.28 \sim 7.25(\mathrm{~m}, 1 \mathrm{H}), 7.24(\mathrm{~d}, J=15 \mathrm{~Hz}, 1 \mathrm{H}), 6.57$ (d, $J=9.5 \mathrm{~Hz}, 1 \mathrm{H}), 6.49(\mathrm{~d}, J=9 \mathrm{~Hz}, 1 \mathrm{H}), 2.14 \sim 2.10(\mathrm{~m}$, $1 \mathrm{H}), 1.71 \sim 1.67(\mathrm{~m}, 1 \mathrm{H}), 0.56(\mathrm{t}, J=7.5 \mathrm{~Hz}, 3 \mathrm{H}) ;{ }^{13} \mathrm{C}$ NMR $\left(125 \mathrm{MHz}, \mathrm{CDCl}_{3}\right) \delta: 184.6,155.5,140.0,136.7$, $133.7,131.6,129.5,128.4,128.1,127.5,127.2,125.4$, 124.9, 121.9, 121.2, 61.9, 35.1, 9.8; HRMS (ESI) calcd for $\mathrm{C}_{18} \mathrm{H}_{16} \mathrm{~N}[\mathrm{M}+\mathrm{H}]^{+}$246.1277, found 246.1285.

$6 \mathrm{a}$-苯基- $6 \mathrm{a} H$-苯并 $[a$ 咔唑 $(\mathbf{2 c})$ ：黄色固体，产率 $83 \%$. m.p. $214 \sim 215{ }^{\circ} \mathrm{C}$; ${ }^{1} \mathrm{H}$ NMR (500 MHz, $\left.\mathrm{CDCl}_{3}\right) \delta$ : $7.86(\mathrm{~d}, J=8.0 \mathrm{~Hz}, 1 \mathrm{H}), 7.71(\mathrm{~d}, J=8.0 \mathrm{~Hz}, 1 \mathrm{H}), 7.37$ (t, $J=7.5 \mathrm{~Hz}, 2 \mathrm{H}), 7.33 \sim 7.28(\mathrm{~m}, 2 \mathrm{H}), 7.22 \sim 7.10(\mathrm{~m}, 7 \mathrm{H})$, $6.76(\mathrm{~d}, J=9.5 \mathrm{~Hz}, 1 \mathrm{H}), 6.72(\mathrm{~d}, J=9.0 \mathrm{~Hz}, 1 \mathrm{H}) ;{ }^{13} \mathrm{C}$ NMR $\left(125 \mathrm{MHz}, \mathrm{CDCl}_{3}\right) \delta: 184.2,155.7,142.5,139.2$, $136.5,131.7,131.5,130.4,129.8,128.8,128.5,127.5$, 127.3, 126.4, 126.1, 125.3, 123.1, 121.6, 64.2; HRMS (ESI) calcd for $\mathrm{C}_{22} \mathrm{H}_{16} \mathrm{~N}[\mathrm{M}+\mathrm{H}]^{+}$294.1277, found 294.1284.

6a-(对甲苯基)- $6 \mathrm{a} H$-苯并 $[a]$ 咔唑(2d): 黄色固体, 产 率 90\%. m.p. 136 137 ${ }^{\circ} \mathrm{C}$; ${ }^{1} \mathrm{H}$ NMR (500 MHz, $\mathrm{CDCl}_{3}$ ) $\delta: 7.87(\mathrm{~d}, J=7.5 \mathrm{~Hz}, 1 \mathrm{H}), 7.72(\mathrm{~d}, J=8.0 \mathrm{~Hz}, 1 \mathrm{H}), 7.39 \sim$ $7.28(\mathrm{~m}, 4 \mathrm{H}), 7.22 \sim 7.16(\mathrm{~m}, 2 \mathrm{H}), 7.02(\mathrm{~d}, J=8.5 \mathrm{~Hz}$, 2H), $6.96(\mathrm{~d}, J=8.0 \mathrm{~Hz}, 2 \mathrm{H}), 6.75$ (d, $J=9.5 \mathrm{~Hz}, 1 \mathrm{H}), 6.70$ $(\mathrm{d}, J=9.5 \mathrm{~Hz}, 1 \mathrm{H}), 2.21(\mathrm{~s}, 3 \mathrm{H}) ;{ }^{13} \mathrm{C} \mathrm{NMR}(125 \mathrm{MHz}$, $\left.\mathrm{CDCl}_{3}\right) \delta: 184.3,155.6,142.6,136.9,136.5,136.0,131.8$, $131.4,130.3,129.5,129.5,128.7,128.3,127.5,126.3$, 126.0, 125.2, 123.0, 121.5, 65.0, 20.9; HRMS (ESI) calcd for $\mathrm{C}_{23} \mathrm{H}_{18} \mathrm{~N}[\mathrm{M}+\mathrm{H}]^{+}$308.1434, found 308.1447.

6a-(4-甲氧基苯基)- $6 \mathrm{a} H$-苯并 $[a]$ 咔唑(2e): 黄色油状 物, 产率 85\%. ${ }^{1} \mathrm{H} \mathrm{NMR}\left(500 \mathrm{MHz}, \mathrm{CDCl}_{3}\right) \delta: 7.87(\mathrm{~d}, J=$ $7.0 \mathrm{~Hz}, 1 \mathrm{H}), 7.73$ (d, J=7.5 Hz, 1H), $7.40 \sim 7.36(\mathrm{~m}, 2 \mathrm{H})$, $7.34 \sim 7.30(\mathrm{~m}, 2 \mathrm{H}), 7.22(\mathrm{t}, J=7.5 \mathrm{~Hz}, 1 \mathrm{H}), 7.19(\mathrm{~d}, J=$ $7.0 \mathrm{~Hz}, 1 \mathrm{H}), 7.04$ (d, $J=9.0 \mathrm{~Hz}, 2 \mathrm{H}), 6.72 \sim 6.67(\mathrm{~m}, 4 \mathrm{H})$, $3.68(\mathrm{~s}, 3 \mathrm{H}) ;{ }^{13} \mathrm{C}$ NMR $\left(125 \mathrm{MHz}, \mathrm{CDCl}_{3}\right) \delta: 184.4,158.7$, $155.5,142.6,136.5,131.8,131.4,130.52,130.2,129.5$, $128.7,128.4,127.5,127.3,126.3,125.3,123.0,121.5$, 114.1, 63.7, 55.1; HRMS (ESI) calcd for $\mathrm{C}_{23} \mathrm{H}_{18} \mathrm{NO}[\mathrm{M}+$ $\mathrm{H}]^{+}$324.1383, found 324.1395.

6a-(4-氟苯基)- $6 \mathrm{a} H$-苯并 $[a]$ 咔唑(2f)：黄色固体，产 率 84\%. m.p. $172 \sim 173{ }^{\circ} \mathrm{C} ;{ }^{1} \mathrm{H}$ NMR $(500 \mathrm{MHz}$, $\left.\mathrm{CDCl}_{3}\right) \delta: 7.85(\mathrm{~d}, J=7.5 \mathrm{~Hz}, 1 \mathrm{H}), 7.73(\mathrm{~d}, J=7.5 \mathrm{~Hz}$, $1 \mathrm{H}), 7.40$ (t, $J=7.5 \mathrm{~Hz}, 2 \mathrm{H}), 7.32$ (t, $J=7.5 \mathrm{~Hz}, 2 \mathrm{H})$, $7.25 \sim 7.20(\mathrm{~m}, 2 \mathrm{H}), 7.10 \sim 7.07(\mathrm{~m}, 2 \mathrm{H}), 6.84(\mathrm{t}, J=8.5$ $\mathrm{Hz}, 2 \mathrm{H}), 6.75(\mathrm{~d}, J=9.5 \mathrm{~Hz}, 1 \mathrm{H}), 6.72$ (d, $J=9.5 \mathrm{~Hz}$, $1 \mathrm{H}) ;{ }^{13} \mathrm{C} \mathrm{NMR}\left(125 \mathrm{MHz}, \mathrm{CDCl}_{3}\right) \delta: 184.1,161.9(\mathrm{~d}$, $J=244.9 \mathrm{~Hz}), 155.6,142.4,136.4,134.48,134.46$, $131.6,131.3,130.1,130.0,128.9,128.6,127.9$ (d, $J=$ 8.1 Hz), 127.6, 126.5, 125.3, 123.1, 121.6, 115.7 (d, $J=$ $20.9 \mathrm{~Hz}$ ), 115.5; HRMS (ESI) calcd for $\mathrm{C}_{22} \mathrm{H}_{15} \mathrm{FN}[\mathrm{M}+$ $\mathrm{H}]^{+}$312.1183, found 312.1203. ${ }^{19} \mathrm{~F}$ NMR (376 MHz, $\left.\mathrm{CDCl}_{3}\right) \delta:-115.24$.

6a-(4-氯苯基)- $6 \mathrm{a} H$-苯并 $[a]$ 咔唑 $(2 \mathrm{~g})$ ：黄色固体，产 率 88\%. m.p. $112 \sim 113{ }^{\circ} \mathrm{C} ;{ }^{1} \mathrm{H}$ NMR $(500 \mathrm{MHz}$, $\left.\mathrm{CDCl}_{3}\right) \delta: 7.84(\mathrm{~d}, J=7.0 \mathrm{~Hz}, 1 \mathrm{H}), 7.72(\mathrm{~d}, J=7.5 \mathrm{~Hz}$, $1 \mathrm{H}), 7.39 \sim 7.36(\mathrm{~m}, 2 \mathrm{H}), 7.31 \sim 7.28(\mathrm{~m}, 2 \mathrm{H}), 7.22 \sim$ $7.17(\mathrm{~m}, 2 \mathrm{H}), 7.10(\mathrm{~d}, J=9.0 \mathrm{~Hz}, 2 \mathrm{H}), 7.03$ (d, $J=8.5$ $\mathrm{Hz}, 2 \mathrm{H}), 6.72$ (d, $J=9.0 \mathrm{~Hz}, 1 \mathrm{H}), 6.69$ (d, $J=9.5 \mathrm{~Hz}$, $1 \mathrm{H}) ;{ }^{13} \mathrm{C} \mathrm{NMR}\left(125 \mathrm{MHz}, \mathrm{CDCl}_{3}\right) \delta 183.8,155.6$, $142.1,137.5,136.3,133.2,131.7,131.0,130.2,130.0$, $128.9,128.9,128.7,127.6,127.6,126.6,125.3,123.0$, 121.7, 63.6; HRMS (ESI) calcd for $\mathrm{C}_{22} \mathrm{H}_{15} \mathrm{CIN}[\mathrm{M}+$ $\mathrm{H}^{+}$328.0888.1183, found 328.0903.

$6 \mathrm{a}$-(邻甲苯基)- $6 \mathrm{a} H$-苯并 $[a$ ]咔唑(2h)：黄色固体，产 率 85\%. m.p. $142 \sim 143{ }^{\circ} \mathrm{C}$; ${ }^{1} \mathrm{H}$ NMR $(600 \mathrm{MHz}$, $\left.\mathrm{CDCl}_{3}\right) \delta: 7.83(\mathrm{~d}, J=7.2 \mathrm{~Hz}, 1 \mathrm{H}), 7.75(\mathrm{~d}, J=7.8 \mathrm{~Hz}$, $1 \mathrm{H}), 7.64$ (d, $J=7.8 \mathrm{~Hz}, 1 \mathrm{H}), 7.38$ (t, $J=7.2 \mathrm{~Hz}, 1 \mathrm{H})$, $7.36 \sim 7.27(\mathrm{~m}, 3 \mathrm{H}), 7.21(\mathrm{t}, J=7.2 \mathrm{~Hz}, 1 \mathrm{H}), 7.16 \sim$ $7.13(\mathrm{~m}, 2 \mathrm{H}), 7.09$ (t, $J=7.2 \mathrm{~Hz}, 1 \mathrm{H}), 6.94$ (d, $J=7.2$ $\mathrm{Hz}, 1 \mathrm{H}), 6.76(\mathrm{~d}, J=9.6 \mathrm{~Hz}, 1 \mathrm{H}), 6.67$ (d, $J=9.6 \mathrm{~Hz}$, $1 \mathrm{H}), 1.91(\mathrm{~s}, 3 \mathrm{H}) ;{ }^{13} \mathrm{C} \mathrm{NMR}\left(150 \mathrm{MHz}, \mathrm{CDCl}_{3}\right) \delta$ : $184.6,156.3,140.7,138.2,136.7,136.3,134.4,132.3$, $131.9,131.0,129.9,128.7,128.3,128.3,127.4,127.2$, 
126.7, 126.2, 125.0, 123.4, 121.3, 64.1, 20.0; HRMS (ESI) calcd for $\mathrm{C}_{23} \mathrm{H}_{18} \mathrm{~N}[\mathrm{M}+\mathrm{H}]^{+}$308.1434, found 308.1454 .

6a-(3,5-二甲基苯基)- $6 \mathrm{a} H$-苯并 $[a]$ 咔唑(2i): 黄色固 体, 产率 83\%. mp 172 173 ${ }^{\circ} \mathrm{C}$; ${ }^{1} \mathrm{H}$ NMR $(500 \mathrm{MHz}$, $\left.\mathrm{CDCl}_{3}\right) \delta: 7.88(\mathrm{~d}, J=7.5 \mathrm{~Hz}, 1 \mathrm{H}), 7.70(\mathrm{~d}, J=7.5 \mathrm{~Hz}$, $1 \mathrm{H}), 7.37 \sim 7.28(\mathrm{~m}, 4 \mathrm{H}), 7.19(\mathrm{~d}, J=8.0 \mathrm{~Hz}, 1 \mathrm{H}), 7.16(\mathrm{~d}$, $J=7.0 \mathrm{~Hz}, 1 \mathrm{H}), 6.77 \sim 6.73(\mathrm{~m}, 4 \mathrm{H}), 6.67(\mathrm{~d}, J=9.0 \mathrm{~Hz}$, $1 \mathrm{H}), 2.15$ (s, 6H); ${ }^{13} \mathrm{C}$ NMR (125 MHz, $\left.\mathrm{CDCl}_{3}\right) \delta: 184.5$, $155.5,142.8,139.3,138.2,136.6,131.9,131.4,130.5$, $129.4,129.1,128.7,128.3,127.5,126.3,125.2,123.8$, 123.0, 121.5, 64.0, 21.4; HRMS (ESI) calcd for $\mathrm{C}_{24} \mathrm{H}_{20} \mathrm{~N}$ $[\mathrm{M}+\mathrm{H}]^{+} 322.1590$, found 322.1600 .

6a-(呋喃-2-基)-6a $H$-苯并 $[a]$ 咔唑(2j): 黄色油状物, 产率 68\%. m.p. 148 $149{ }^{\circ} \mathrm{C}$; ${ }^{1} \mathrm{H}$ NMR $(500 \mathrm{MHz}$, $\left.\mathrm{CDCl}_{3}\right) \delta: 7.93(\mathrm{~d}, J=7.5 \mathrm{~Hz}, 1 \mathrm{H}), 7.71(\mathrm{~d}, J=7.5 \mathrm{~Hz}$, $1 \mathrm{H}), 7.51(\mathrm{~d}, J=7.5 \mathrm{~Hz}, 1 \mathrm{H}), 7.42 \sim 7.38(\mathrm{~m}, 2 \mathrm{H}), 7.34$ (t, $J=7.5 \mathrm{~Hz}, 1 \mathrm{H}), 7.25$ (t, $J=8.0 \mathrm{~Hz}, 1 \mathrm{H}), 7.21 \sim 7.18$ $(\mathrm{m}, 2 \mathrm{H}), 6.64 \sim 6.59(\mathrm{~m}, 2 \mathrm{H}), 6.12 \sim 6.11(\mathrm{~m}, 1 \mathrm{H}), 5.96$ $(\mathrm{d}, J=3.0 \mathrm{~Hz}, 1 \mathrm{H}) ;{ }^{13} \mathrm{C}$ NMR $\left(125 \mathrm{MHz}, \mathrm{CDCl}_{3}\right) \delta$ : 180.5, 155.6, 151.0, 142.7, 138.6, 136.5, 131.6, 130.0, $129.7,129.4,128.9,128.8,127.7,126.2,125.2,123.3$, 121.6, 110.1, 106.0, 60.5; HRMS (ESI) calcd for $\mathrm{C}_{20} \mathrm{H}_{15} \mathrm{NO}[\mathrm{M}+\mathrm{H}]^{+}$284.1070, found 284.1087.

6a-(䒺-2-基)-6a $H$-苯并 $[a]$ 咔唑(2k): 黄色油状物, 产率 $89 \% .{ }^{1} \mathrm{H}$ NMR $\left(500 \mathrm{MHz}, \mathrm{CDCl}_{3}\right) \delta: 7.87$ (d, $J=7.5$ $\mathrm{Hz}, 1 \mathrm{H}), 7.77 \sim 7.76(\mathrm{~m}, 2 \mathrm{H}), 7.71 \sim 7.67(\mathrm{~m}, 2 \mathrm{H}), 7.58(\mathrm{~d}$, $J=9.0 \mathrm{~Hz}, 1 \mathrm{H}), 7.41 \sim 7.36(\mathrm{~m}, 3 \mathrm{H}), 7.35 \sim 7.32(\mathrm{~m}, 2 \mathrm{H})$, $7.28(\mathrm{~d}, J=7.5 \mathrm{~Hz}, 1 \mathrm{H}), 7.19$ (t, $J=8.0 \mathrm{~Hz}, 2 \mathrm{H}), 7.00 \sim$ $7.98(\mathrm{~m}, 1 \mathrm{H}), 6.86(\mathrm{~d}, J=9.5 \mathrm{~Hz}, 1 \mathrm{H}), 6.80(\mathrm{~d}, J=9.5 \mathrm{~Hz}$, $1 \mathrm{H}) ;{ }^{13} \mathrm{C}$ NMR $\left(125 \mathrm{MHz}, \mathrm{CDCl}_{3}\right) \delta 184.3,155.7,142.5$, $136.6,136.4,133.4,132.4,131.5,131.4,130.1,128.8$, $128.6,128.5,127.9,127.6,127.4,126.5,126.1,126.0$, 125.2, 125.1, 124.2, 123.2, 121.6, 64.2; HRMS (ESI) calcd for $\mathrm{C}_{26} \mathrm{H}_{18} \mathrm{~N}[\mathrm{M}+\mathrm{H}]^{+}$344.1434, found 344.1450.

5,6a-二-对甲苯基- $6 \mathrm{a} H$-苯并 $[a]$ 咔唑(21): 黄色固体, 产率 91\%. m.p. $187 \sim 188{ }^{\circ} \mathrm{C}$; ${ }^{1} \mathrm{H}$ NMR $(500 \mathrm{MHz}$, $\left.\mathrm{CDCl}_{3}\right) \delta: 7.92 \sim 7.89(\mathrm{~m}, 1 \mathrm{H}), 7.75(\mathrm{~d}, J=7.5 \mathrm{~Hz}, 1 \mathrm{H})$, $7.37(\mathrm{t}, J=7.5 \mathrm{~Hz}, 1 \mathrm{H}), 7.33 \sim 7.31(\mathrm{~m}, 5 \mathrm{H}), 7.25(\mathrm{~d}, J=$ $7.5 \mathrm{~Hz}, 2 \mathrm{H}), 7.19(\mathrm{t}, J=7.5 \mathrm{~Hz}, 1 \mathrm{H}), 7.09 \sim 7.07(\mathrm{~m}, 3 \mathrm{H})$, $7.01(\mathrm{~d}, J=8.0 \mathrm{~Hz}, 1 \mathrm{H}), 6.67(\mathrm{~s}, 1 \mathrm{H}), 2.43(\mathrm{~s}, 3 \mathrm{H}), 2.24$ (s, $3 \mathrm{H}) ;{ }^{13} \mathrm{C}$ NMR $\left(125 \mathrm{MHz}, \mathrm{CDCl}_{3}\right) \delta: 184.2,155.8,143.0$, $141.7,137.7,137.2,136.9,136.5,136.4,131.1,130.5$, $129.6,129.6,129.1,128.7,128.7,128.3,127.4,126.4$, 126.0, 125.38, 123.1, 121.4, 63.8, 21.3, 20.9; HRMS (ESI) calcd for $\mathrm{C}_{30} \mathrm{H}_{24} \mathrm{~N}[\mathrm{M}+\mathrm{H}]^{+}$398.1903, found 398.1918.

5,6a-二-(4-氯苯基)-6a $H$-苯并 $[a]$ 咔唑(2m): 黄色油 状物, 产率 85\%. ${ }^{1} \mathrm{H}$ NMR $\left(500 \mathrm{MHz}, \mathrm{CDCl}_{3}\right) \delta:$ 7.92 $7.90(\mathrm{~m}, 1 \mathrm{H}), 7.77(\mathrm{~d}, J=8.0 \mathrm{~Hz}, 1 \mathrm{H}), 7.42 \sim 7.35(\mathrm{~m}$, $5 \mathrm{H}), 7.32(\mathrm{~d}, J=7.5 \mathrm{~Hz}, 1 \mathrm{H}), 7.23(\mathrm{t}, J=7.0 \mathrm{~Hz}, 1 \mathrm{H})$, $7.16 \sim 7.13(\mathrm{~m}, 4 \mathrm{H}), 7.05 \sim 7.03(\mathrm{~m}, 1 \mathrm{H}), 6.89(\mathrm{t}, J=8.5$ $\mathrm{Hz}, 2 \mathrm{H}), 6.65(\mathrm{~s}, 1 \mathrm{H}) ;{ }^{13} \mathrm{C}$ NMR $\left(125 \mathrm{MHz}, \mathrm{CDCl}_{3}\right) \delta$ : $183.6,155.7,142.5,141.4,136.8,131.3,130.5,130.4$, $130.3,129.7,129.1,128.7,127.8,127.8,127.3,126.6$, $125.5,123.2,121.7,115.9,115.7,115.6,115.4,63.3$; HRMS (ESI) calcd for $\mathrm{C}_{28} \mathrm{H}_{18} \mathrm{Cl}_{2} \mathrm{~N}[\mathrm{M}+\mathrm{H}]^{+} 438.0811$, found 438.0825 .

6a-甲基-5-苯基-6a $H$-苯并 $[a]$ 咔唑(2n): 黄色油状物, 产率 94\%. ${ }^{1} \mathrm{H}$ NMR $\left(600 \mathrm{MHz}, \mathrm{CDCl}_{3}\right) \delta: 8.10 \sim 8.08(\mathrm{~m}$, 1H), $7.75(\mathrm{~d}, J=7.8 \mathrm{~Hz}, 1 \mathrm{H}), 7.47(\mathrm{~d}, J=7.2 \mathrm{~Hz}, 1 \mathrm{H})$, $7.44 \sim 7.39(\mathrm{~m}, 6 \mathrm{H}), 7.34(\mathrm{~d}, J=6.6 \mathrm{~Hz}, 2 \mathrm{H}), 7.28 \sim 7.25$ (m, 1H), $7.14(\mathrm{~d}, J=7.2 \mathrm{~Hz}, 1 \mathrm{H}), 6.50(\mathrm{~s}, 1 \mathrm{H}), 1.57$ (s, $3 \mathrm{H}) ;{ }^{13} \mathrm{C}$ NMR $\left(150 \mathrm{MHz}, \mathrm{CDCl}_{3}\right) \delta: 185.3,154.8,142.1$, $139.5,139.1,137.1,132.2,131.3,129.3,129.0,128.5$, $128.4,128.2,127.7,127.3,125.8,125.4,121.7,121.5$, 56.9, 27.5; HRMS (ESI) calcd for $\mathrm{C}_{23} \mathrm{H}_{18} \mathrm{~N}[\mathrm{M}+\mathrm{H}]^{+}$ 308.1434 , found 308.1449 .

5-(4-氯苯基)-6a-甲基- $6 \mathrm{a} H$-苯并 $[a]$ 咔唑(2o)：黄色 油状物, 产率 96\%. ${ }^{1} \mathrm{H}$ NMR (600 MHz, $\left.\mathrm{CDCl}_{3}\right) \delta: 8.09 \sim$ $8.08(\mathrm{~m}, 1 \mathrm{H}), 7.74(\mathrm{~d}, J=7.8 \mathrm{~Hz}, 1 \mathrm{H}), 7.48 \sim 7.39(\mathrm{~m}$, $6 \mathrm{H}), 7.28 \sim 7.26(\mathrm{~m}, 3 \mathrm{H}), 7.09 \sim 7.08(\mathrm{~m}, 1 \mathrm{H}), 6.49(\mathrm{~s}$, 1H), 1.55 (s, $3 \mathrm{H}) ;{ }^{13} \mathrm{C}$ NMR (150 MHz, $\left.\mathrm{CDCl}_{3}\right) \delta: 184.9$, $154.8,141.9,138.1,138.0,136.7,133.7,132.6,131.4$, $130.3,129.3,128.7,128.6,128.3,127.1,125.9,125.5$, 121.7, 121.6, 56.9, 27.4; HRMS (ESI) calcd for $\mathrm{C}_{23} \mathrm{H}_{17} \mathrm{ClN}$ $[\mathrm{M}+\mathrm{H}]^{+}$342.1044, found 342.1062 .

8,9-二甲氧基-6a-甲基- $6 \mathrm{a} H$-苯并 $[a]$ 咔唑(2p)：黄色 油状物, 产率 74\%. ${ }^{1} \mathrm{H}$ NMR $\left(500 \mathrm{MHz}, \mathrm{CDCl}_{3}\right) \delta: 7.96$ (d, $J=7.5 \mathrm{~Hz}, 1 \mathrm{H}), 7.42$ (t, $J=6.0 \mathrm{~Hz}, 1 \mathrm{H}), 7.37$ (t, $J=6.5$ $\mathrm{Hz}, 1 \mathrm{H}), 7.31$ (s, 1H), 7.21 (d, $J=7.0 \mathrm{~Hz}, 1 \mathrm{H}), 6.98$ (s, $1 \mathrm{H}), 6.51(\mathrm{~d}, J=9.5 \mathrm{~Hz}, 1 \mathrm{H}), 6.44(\mathrm{~d}, J=9.5 \mathrm{~Hz}, 1 \mathrm{H}), 3.96$ (s, 3H), 3.94 (s, 3H), 1.45 (s, 3H); ${ }^{13} \mathrm{C}$ NMR (125 MHz, $\left.\mathrm{CDCl}_{3}\right) \delta: 184.3,149.2,147.8,147.7,136.4,133.9,133.6$, $131.2,129.2,128.4,127.5,126.9,124.9,105.4,105.1$, $57.5,56.5,56.2,28.4$; HRMS (ESI) calcd for $\mathrm{C}_{19} \mathrm{H}_{18} \mathrm{NO}_{2}$ $[\mathrm{M}+\mathrm{H}]^{+}$292.1332, found 292.1345.

$6 \mathrm{a}$-甲基- $6 \mathrm{a} H$-苯并 $[a][1,3]$ 二氧杂环戊烯并 $[4,5-h]$ 咔 唑(2q)：黄色油状物, 产率 $48 \%$. ${ }^{1} \mathrm{H}$ NMR $(500 \mathrm{MHz}$, $\left.\mathrm{CDCl}_{3}\right) \delta: 7.94(\mathrm{~d}, J=8.0 \mathrm{~Hz}, 1 \mathrm{H}), 7.42(\mathrm{t}, J=7.5 \mathrm{~Hz}, 1 \mathrm{H})$, $7.36(\mathrm{t}, J=6.0 \mathrm{~Hz}, 1 \mathrm{H}), 7.21 \sim 7.19(\mathrm{~m}, 2 \mathrm{H}), 6.91(\mathrm{~s}, 1 \mathrm{H})$, 
$6.47(\mathrm{~d}, J=9.0 \mathrm{~Hz}, 1 \mathrm{H}), 6.43(\mathrm{~d}, J=9.5 \mathrm{~Hz}, 1 \mathrm{H}), 6.00(\mathrm{~s}$, 2H), 1.43 (s, 3H); ${ }^{13} \mathrm{C}$ NMR (125 MHz, $\left.\mathrm{CDCl}_{3}\right) \delta: 184.6$, $148.9,147.5,146.1,136.4,135.5,133.8,131.3,129.2$, 128.5, 127.5, 127.0, 124.9, 103.3, 102.6, 101.5, 57.2, 28.3; HRMS (ESI) calcd for $\mathrm{C}_{18} \mathrm{H}_{14} \mathrm{NO}_{2}[\mathrm{M}+\mathrm{H}]^{+}$276.1019, found 276.1032 .

6-溴-6a-苯基-6,6a-二氢-5H-苯并 $[a$ 咔唑-5-醇 (3): 黄色固体, 产率 86\%, m.p. 162 163 ${ }^{\circ} \mathrm{C} .{ }^{1} \mathrm{H}$ NMR $(500$ $\left.\mathrm{MHz}, \mathrm{CDCl}_{3}\right) \delta: 8.39 \sim 8.37(\mathrm{~m}, 1 \mathrm{H}), 7.69(\mathrm{~d}, J=8.0 \mathrm{~Hz}$, $1 \mathrm{H}), 7.58 \sim 7.49(\mathrm{~m}, 4 \mathrm{H}), 7.36(\mathrm{t}, J=7.0 \mathrm{~Hz}, 3 \mathrm{H}), 7.26 \sim$ $7.19(\mathrm{~m}, 4 \mathrm{H}), 5.53(\mathrm{~d}, J=2.0 \mathrm{~Hz}, 1 \mathrm{H}), 5.29$ (d, $J=8.5 \mathrm{~Hz}$, $1 \mathrm{H}) ;{ }^{13} \mathrm{C}$ NMR $\left(125 \mathrm{MHz}, \mathrm{CDCl}_{3}\right) \delta: 176.7,154.6,143.2$, $138.8,137.4,132.5,131.1,129.8,129.6,129.1,128.8$, 128.4, 126.9, 126.5, 125.4, 122.6, 121.6, 74.4, 63.7, 53.7. LCMS (ESI) calcd for $\mathrm{C}_{22} \mathrm{H}_{17} \mathrm{BrNO}[\mathrm{M}+\mathrm{H}]^{+} 390.0488$, found 390.0505 .

$10 \mathrm{~b}$ - 苯基- $10 \mathrm{~b}, 10 \mathrm{c}$-二氢- $1 \mathrm{a} H$-苯并 $[a]$ 环氧乙烯并 [2,3-c] 咔唑 (4): 白色固体, 产率 92\%; m.p. 163 164 ${ }^{\circ} \mathrm{C}$. ${ }^{1} \mathrm{H}$ NMR $\left(500 \mathrm{MHz}, \mathrm{CDCl}_{3}\right) \delta: 8.08(\mathrm{~d}, J=7.0 \mathrm{~Hz}, 1 \mathrm{H})$, $7.70(\mathrm{~d}, J=8.0 \mathrm{~Hz}, 1 \mathrm{H}), 7.61 \sim 7.48(\mathrm{~m}, 4 \mathrm{H}), 7.36(\mathrm{t}, J=$ $6.5 \mathrm{~Hz}, 1 \mathrm{H}), 7.26 \sim 7.17(\mathrm{~m}, 5 \mathrm{H}), 7.11(\mathrm{t}, J=7.0 \mathrm{~Hz}, 1 \mathrm{H})$, $4.33(\mathrm{~d}, J=3.5 \mathrm{~Hz}, 1 \mathrm{H}), 4.05(\mathrm{~d}, J=3.5 \mathrm{~Hz}, 1 \mathrm{H}) ;{ }^{13} \mathrm{C}$ NMR $\left(125 \mathrm{MHz}, \mathrm{CDCl}_{3}\right) \delta: 181.5,154.8,143.1,139.8$, $133.9,131.6,131.4,131.0,130.2,128.8,128.8,127.1$, 126.6, 126.5, 125.7, 122.8, 121.7, 63.2, 59.6, 52.6. LCMS (ESI) calcd for $\mathrm{C}_{22} \mathrm{H}_{16} \mathrm{NO} 310.1226[\mathrm{M}+\mathrm{H}]^{+}$, found 310.1226

辅助材料(Supporting Information) 化合物 $\mathbf{2 a} \sim \mathbf{2 q}, \mathbf{3}$ 和 4 的 ${ }^{1} \mathrm{H}$ NMR 和 ${ }^{13} \mathrm{C} \mathrm{NMR}$ 原始谱图. 这些材料可以免 费从本刊网站(http://sioc-journal.cn/)上下载.

\section{References}

[1] (a) Zheng, C.; You, S.-L. ACS Cent. Sci. 2021, 7, 432.

(b) Sharma, U. K.; Ranjan, P.; Van der Eycken, E. V.; You, S.-L. Chem. Soc. Rev. 2020, 49, 8721 .

(c) Huang, G.; Yin, B. Adv. Synth. Catal. 2019, 361, 405.

(d) Wu, W.-T.; Zhang, L.; You, S.-L. Chem. Soc. Rev. 2016, 45, 1570 .

(e) Zheng, C.; You, S.-L. Chem 2016, 1, 830 .

(f) Zhuo, C.-X.; Zheng, C.; You, S.-L. Acc. Chem. Res. 2014, 47, 2558

(g) Zhuo, C.-X.; Zhang, W.; You, S.-L. Angew. Chem., Int. Ed. 2012, 51,1266

(h) Li, K.; Bai, L.; Luan, X. Chin. J. Org. Chem. 2019, 39, 2211 (in Chinese).

(李锟雨, 白璐, 奕新军, 2019, 39, 2211.)

(i) Yan, Q.; Fan, R.; Liu, B.; Su, S.; Wang, B.; Yao, T.; Tan, J. Chin. J. Org. Chem. 2021, 41, 455 (in Chinese).

(甩强, 范荣, 刘斌斌, 苏帅松, 王勃, 姚团利, 谭嘉靖, 有机化 学, 2021, 41, 455.)

[2] (a) Bai, Y.; Liu, A.; Wu, X.-X.; Chen, S.; Wang, J. J. Org. Chem.
$2020,85,6687$.

(b) Hu, J.; Pan, S.; Zhu, S.; Yu, P.; Xu, R; Zhong, G.; Zeng, X. J. Org. Chem. 2020, 85, 7896.

(c) Wu, X.-X.; Liu, A.; Mou, M.; Chen, H.; Chen, S. J. Org. Chem. 2018, 83, 14181.

(d) Fan, L.; Liu, J.; Bai, L.; Wang, Y.; Luan, X. Angew. Chem., Int. Ed. 2017, 56, 14257.

(e) Luo, L.; Zheng, H.; Liu, J.; Wang, H.; Wang, Y.; Luan, X. Org. Lett. 2016, 18, 2082.

(f) Nemoto, T., Nozaki, T.; Yoshida, M.; Hamada, Y. Adv. Synth. Catal. 2013, 355, 2693.

(g) Nemoto, T.; Zhao, Z. D.; Yokosaka, T.; Suzuki, Y.; Wu, R.; Hamada, Y. Angew. Chem., Int. Ed. 2013, 52, 2217.

(h) Yoshida, M.; Nemoto, T.; Zhao, Z.; Ishige, Y.; Hamada, Y. Tetrahedron: Asymmetry 2012, 23, 859.

(i) Rousseaux, S.; Fortanet, J. G.; Sanchez, M. A. D. A.; Buchwald, S. L. J. Am. Chem. Soc. 2011, 133, 9282.

(j) Wu, Q.-F.; Liu, W.-B.; Zhuo, C.-X.; Rong, Z.-Q.; Ye, K.-Y.; You, S.-L. Angew. Chem., Int. Ed. 2011, 50, 4455.

(k) Nemoto, T.; Ishige, Y.; Yoshida, M.; Kohno, Y.; Kanematsu, M.; Hamada, Y. Org. Lett. 2010, 12, 5020.

[3] (a) Bedford, R. B.; Fey, N.; Haddow, M. F.; Sankey, R. F. Chem. Commun. 2011, 47, 3649.

(b) Bedford, R. B.; Butts, C. P.; Haddow, M. F.; Osborne, R.; Sankey, R. F. Chem. Commun. 2009, 4832.

[4] (a) Fu, Z.; Zhu, J.; Guo, S.; Lin, A. Chem. Commun. 2021, 57 , 1262 .

(b) Yamaguchi, M.; Fujiwara, S.; Manabe, K. Org. Lett. 2019, 21, 6972.

(c) Ding, L.; Gao, R.-D.; You, S. L. Chem. Eur. J. 2019, 25, 4330.

(d) Fang, X.; Li, Q.; Shi, R.; Yao, H.; Lin, A. Org. Lett. 2018, 20 , 6084 .

(e) Trost, B. M.; Bai, W.-J.; Hohn, C.; Bai, Y.; Cregg, J. J. Am Chem. Soc. 2018, 140, 6710.

(f) Gao, R.-D.; Ding, L.; Zheng, C.; Dai, L.-X.; You, S.-L. Org. Lett. 2018, 20, 748.

(g) Gao, S.; Wu, Z.; Fang, X.; Lin, A.; Yao, H. Org. Lett. 2016, 18 , 3906.

(h) Gao, R.-D.; Xu, Q.-L.; Zhang, B.; Gu, Y.; Dai, L.-X.; You, S.-L. Chem. Eur. J. 2016, 22, 11601.

(i) Zhang, H.; Hu, R.-B.; Liu, N.; Li, S.-X.; Yang, S.-D. Org. Lett. 2016, 18,28

(j) Zhuo, C.-X.; Cheng, Q.; Liu, W.-B.; Zhao, Q.; You, S.-L. Angew. Chem., Int. Ed. 2015, 54, 8475 .

(k) Gao, R.-D.; Liu, C.; Dai, L.-X.; Zhang, W.; You, S.-L. Org. Lett. 2014, 16, 3919.

(1) Yang, Z.-P.; Zhuo, C.-X.; You, S.-L. Adv. Synth. Catal. 2014 356,1731

(m) Zhang, X.; Han, L.; You, S.-L. Chem. Sci. 2014, 5, 1059.

(n) Zhang, X.; Liu, W.-B.; Wu, Q.-F.; You, S.-L. Org. Lett. 2013, 15,3746 .

(o) Montgomery, T. D.; Zhu, Y.; Kagawa, N.; Rawal, V. H. Org Lett. 2013, 15, 1140 .

(p) Wu, K.-J.; Dai, L.-X.; You, S.-L. Org. Lett. 2012, 14, 3772.

(q) Wu, Q.-F.; Zheng, C.; You, S.-L. Angew. Chem., Int. Ed. 2012 51,1680 .

(r) Wu, K.-J.; Dai, L.-X.; You, S.-L. Org. Lett. 2012, 14, 3772.

(s) Trost, B. M.; Quancard, J. J. Am. Chem. Soc. 2006, 128, 6314

(t) Kimura, M.; Futamata, M.; Mukai, R.; Tamaru, Y. J. Am. Chem. Soc. 2005, 127, 4592 .

[5] (a) Liang, R.-X.; Song, L.-J.; Lu, J.-B.; Xu, W.-Y.; Ding, C.; Jia Y.-X. Angew. Chem., Int. Ed. 2021, 60, 7412.

(b) Zhou, B.; Wang, H.; Cao, Z.-Y.; Zhu, J.-W.; Liang, R.-X.; Jia, Y.-X. Nat. Commun. 2020, 11, 4380.

(c) Liang, R.-X.; Wang, K.; Wu, Q.; Sheng, W.-J.; Jia, Y.-X. Organometallics 2019, 38, 3927.

(d) Weng, J.-Q.; Xing, L.-L.; Hou, W.-R.; Liang, R.-X.; Jia, Y.-X. Org. Chem. Front. 2019, 6, 1577. 
(e) Marchese, A. D.; Lind, F.; MahonÁ, E.; Yoon, H.; Lautens, M. Angew. Chem., Int. Ed. 2019, 58, 5095.

(f) Liang, R.-X.; Wang, K.; Song, L.-J.; Sheng, W.-J.; Jia, Y.-X. RSC Adv. 2019, 9, 13959.

(g) Shen, C.; Zeidan, N.; Wu, Q.; Breuers, C. B. J.; Liu, R.-R.; Jia, Y.-X.; Lautens, M. Chem. Sci. 2019, 10, 3118.

(h) Li, X.; Zhou, B.; Yang, R.-Z.; Yang, F.-M.; Liang, R.-X.; Liu, R.-R.; Jia, Y.-X. J. Am. Chem. Soc. 2018, 140, 13945.

(i) Zeidan, N.; Beisel, T.; Ross, R.; Lautens, M. Org. Lett. 2018, 20, 7332 .

(j) Yang, P.; You, S.-L. Org. Lett. 2018, 20, 7684.

(k) Liang, R.-X.; Yang, R.-Z.; Liu, R.-R.; Jia, Y.-X. Org. Chem. Front. 2018, 5, 1840.

(1) Wang, Y.; Liu, R.; Gao, J; Jia, Y. Chin. J. Org. Chem. 2017, 37, 691 (in Chinese).

(王永刚，刘人荣，高建荣，贾义霞，有机化学，2017，37, 691.) (m) Qin, X.; Lee, M. W. Y.; Zhou, J. S. Angew. Chem., Int. Ed. 2017, 56, 12723.

(n) Liu, R.-R.; Xu, Y.; Liang, R.-X.; Xiang, B.; Xie, H.-J., Gao, J.-R.; Jia, Y.-X. Org. Biomol. Chem. 2017, 15, 2711.

(o) Liu, R.-R.; Wang, Y.-G.; Li, Y.-L.; Huang, B.-B.; Liang, R.-X.; Jia, Y.-X. Angew. Chem., Int. Ed. 2017, 56, 7475.

(p) Petrone, D. A.; Kondo, M.; Zeidan, N.; Lautens, M. Chem. Eur. J. 2016, 22, 5684 .

(q) Chen, S.; Wu, X.-X.; Wang, J.; Hao, X.-H.; Xia, Y.; Shen, Y.; Jing, H.; Liang, Y.-M. Org. Lett. 2016, 18, 4016.

(r) Liu, R.-R.; Xu, T.-F.; Wang, Y.-G.; Xiang, B.; Gao, J.-R.; Jia,
Y.-X. Chem. Commun. 2016, 52, 13664.

(s) Douki, K.; Ono, H.; Taniguchi, T.; Shimokawa, J.; Kitamura, M.; Fukuyama, T. J. Am. Chem. Soc. 2016, 138, 14578.

(t) Gao, S.; Yang, C.; Huang, Y.; Zhao, L.; Wu, X.; Yao, H.; Lin, A. Org. Biomol. Chem. 2016, 14, 840.

(u) Shen, C.; Liu, R.-R.; Fan, R.-J.; Li, Y.-L.; Xu, T.-F.; Gao, J.-R.; Jia, Y.-X. J. Am. Chem. Soc. 2015, 137, 4936;

(v) Petrone, D. A.; Yen, A.; Zeidan, N.; Lautens, M. Org. Lett. 2015, 17, 4838.

(w) Zhao, L.; Li, Z.; Chang, L.; Xu, J.; Yao, H.; Wu, X. Org. Lett. 2012, 14, 2066.

[6] (a) Ma, D.; Cai, Q. Acc. Chem. Res. 2008, 41, 1450.

(b) Hassan, J.; Sévignon, M.; Gozzi, C.; Schulz, E.; Lemaire, M. Chem. Rev. 2002, 102, 1359.

[7] (a) Gao, D.; Parvez, M.; Back, T. G. Chem. Eur. J. 2010, 16, 14281. (b) Bernini, R.; Cacchi, S.; Fabrizi, G.; Filisti, E.; Sferrazza, A. Synlett 2009, 1480

(c) Yan, S.; Wu, H.; Wu, N.; Jiang, Y. Synlett 2007, 2699.

(d) Osuka, A.; Mori, Y.; Suzuki, H. Chem. Lett. 1982, 12, 2031.

[8] Fortanet, J. G.; Kessler, F.; Buchwald, S. L. J. Am. Chem. Soc. 2009, 131, 6676

[9] (a) Liang, R.-X.; Zhong, C.; Liu, Z.-H.; Yang, M.; Tang, H.-W.; Chen, J.-F.; Yang, Y.-F.; Jia, Y.-X. ACS Catal. 2021, 11, 1827.

(b) Xu, H.-B.; Zhu, Y.-Y.; Dong, L. J. Org. Chem. 2019, 84, 16286.

(c) Jana, N.; Zhou, F.; Driver, T. G. J. Am. Chem. Soc. 2015, 137, 6738 .

(d) Kong, C.; Driver, T. G. Org. Lett. 2015, 17, 802. 